\title{
Cryogenic magnetic coil and superconducting magnetic shield for neutron electric dipole moment searches
}

\author{
S. Slutskya , C. M. Swank ${ }^{a}$, A. Biswas ${ }^{a}$, R. Carr ${ }^{\mathrm{a}}$, J. Escribano ${ }^{\mathrm{a}, \mathrm{b}}$, B. W. Filippone ${ }^{\mathrm{a}}$, W. C. \\ Griffith $^{\mathrm{a}, \mathrm{c}}$, M. Mendenhall ${ }^{\mathrm{a}, \mathrm{d}}$, N. Nouri ${ }^{\mathrm{e}}$, C. Osthelder ${ }^{\mathrm{a}}$, A. Pérez Galván ${ }^{\mathrm{a}, \mathrm{f}}$, R. Picker ${ }^{\mathrm{a}, \mathrm{g}}, \mathrm{B}$. \\ Plastere $^{\mathrm{e}}$ \\ ${ }^{a}$ Department of Physics, Math and Astronomy, California Institute of Technology, Pasadena, CA 91125 USA \\ ${ }^{b}$ Xamarin, San Francisco, CA 94111 USA \\ ${ }^{c}$ Department of Physics and Astronomy, University of Sussex, Brighton, BN1 9RH United Kingdom \\ ${ }^{d}$ National Institute of Standards and Technology, Gaithersburg, MD 20899 USA \\ ${ }^{e}$ Department of Physics and Astronomy, University of Kentucky, Lexington, KY 40506 USA \\ ${ }^{f}$ Vertex Pharmaceuticals, 11010 Torreyana Rd., San Diego, CA 92121 USA \\ ${ }^{g}$ TRIUMF, Vancouver, BC V6T 2A3, Canada
}

\begin{abstract}
A magnetic coil operated at cryogenic temperatures is used to produce spatial, relative field gradients below $6 \mathrm{ppm} / \mathrm{cm}$, stable for several hours. The apparatus is a prototype of the magnetic components for a neutron electric dipole moment (nEDM) search, which will take place at the Spallation Neutron Source (SNS) at Oak Ridge National Laboratory using ultra-cold neutrons (UCN). That search requires a uniform magnetic field to mitigate systematic effects and obtain long polarization lifetimes for neutron spin precession measurements. This paper details upgrades to a previously described apparatus [1], particularly the introduction of super-conducting magnetic shielding and the associated cryogenic apparatus. The magnetic gradients observed are sufficiently low for the nEDM search at SNS.
\end{abstract}

\section{Introduction}

The existence of a permanent electric-dipole-moment (EDM) on a subatomic scale would violate both parity $(\mathrm{P})$ and time $(\mathrm{T})$ symmetries, and would be a signature of physics beyond the Standard Model [2]. Additionally, with CPT symmetry, such an EDM would also violate the combined charge and parity (CP) symmetry. The amount of $\mathrm{CP}$ violation currently observed in meson decay cannot explain the baryonic matter/anti-matter asymmetry in the observed universe, within the Sakharov criteria [3]. Therefore, a larger source of CP violation is anticipated. With the expected precision of the next generation of competitive EDM searches, a positive or null measurement will have broad theoretical consequences [4, 5, 6].

Of the species available to probe EDMs, the neutron has several advantages. Relative to atoms or charged particles, the neutron can be considered simple to understand and manipulate. It has no electrons to shield or enhance the effect of an EDM, mitigating errors that can arise from theoretical predictions. Its trajectory is not affected by uniform electric fields, and it can be trapped in a material bottle and observed for periods only limited by its intrinsic lifetime. Currently, the most precise nEDM measurement was made using ultracold neutrons (UCN) by the Sussex/RAL/ILL nEDM experiment, setting a limit of $3.0 \times 10^{-26} \mathrm{e}-\mathrm{cm}$ [7]. Among the 
next generation of EDM experiments, the nEDM search at the SNS [8], based on the concepts discussed in [9], is possibly the most ambitious of all, with a design sensitivity of $d_{n} \lesssim 3 \times 10^{-28}$ e-cm.

While the Sussex/RAL/ILL experiment was statistically limited, the largest systematic uncertainty was due to the so-called "geometric phase" [10]. When a particle's spin precession frequency is measured in the presence of a magnetic field with a spatial gradient, there is a frequency shift proportional to both the linear magnetic field gradients and to the applied electric field; this can mimic the expected EDM signal. It is therefore critical to fully understand the magnetic environment of the nEDM measurement apparatus and to minimize magnetic impurities and non-uniformities. This paper will discuss magnetic measurements taken with a prototype apparatus designed to replicate the magnetic coils and shielding of the nEDM at SNS experiment at half-scale and evaluate the magnetic adequacy of the designs.

\section{Experimental apparatus}

\subsection{Summary of the $n E D M$ apparatus at the SNS}

In the SNS nEDM experiment, neutrons will be generated at the mercury spallation source and moderated to low temperatures, $\sim 20-30 \mathrm{~K}$. The cold neutrons will be spin-polarized with a supermirror polarizer and then guided into the cryogenic apparatus, where they will illuminate two cells filled with isotopically pure ${ }^{4} \mathrm{He}$. The two cells will be held at a temperature of $\sim 450$ $\mathrm{mK}$ by a large dilution refrigerator. Neutrons with a wavelength of 8.9 Angstroms can interact with the superfluid via phonon emission and down-scatter to an energy of $\sim 0-200 \mathrm{neV}$ [11, 12]. These neutrons will be moving so slowly that they become trapped between the walls of the cell, which are coated with deuterated polystyrene. The neutrons that become trapped are considered UCN.

Trapped UCN will be subjected to a magnetic holding field, $B_{0} \sim 3 \mu \mathrm{T}$, to maintain polarization. A strong electric field, $E$, will be applied to probe the EDM, the direction of which can be reversed to control for systematics. A $\pi / 2$ pulse will rotate the UCN spins perpendicular to the holding field. The UCN spins will then precess according to their Larmor frequency, $\omega_{n}$ :

$$
\omega_{n}=-2\left(\mu_{n} B_{0} \pm d_{n} E\right) / \hbar
$$

where $\mu_{n}$ and $d_{n}$ are the neutron magnetic and electric dipole moments, respectively, and $\hbar$ is Planck's constant. The sign of the EDM term depends on the direction of the applied field. Thus, a neutron spin-precession frequency shift proportional to $E$ indicates a non-zero nEDM.

The frequency shift will be extracted by the spin-dependent interaction with ${ }^{3} \mathrm{He}$ [13]. Polarized ${ }^{3} \mathrm{He}$ will be injected at the time of measurement, will be subjected to a $\pi / 2$ pulse to align the spins with those of the UCN, and will diffuse throughout the cell to co-habit with the neutrons. The ${ }^{3} \mathrm{He}$ EDM is known to be negligible compared to the neutron EDM, so the ${ }^{3} \mathrm{He}$ spin can be considered to not precess under the applied electric field [14]. A neutron and ${ }^{3} \mathrm{He}$ in proximity and with opposing spins can react to form a proton and triton with $764 \mathrm{keV}$. Charged decay products will cause the superfluid helium-II to scintillate, and the scintillations are observed to form the signal. This signal will oscillate in time as the neutron spins precess from aligned to anti-aligned with the ${ }^{3} \mathrm{He}$, and it will be maximized if the neutron and ${ }^{3} \mathrm{He}$ spins remain in the same plane. Thus, a uniform magnetic field is necessary to maximize the neutron and ${ }^{3} \mathrm{He}$ transverse coherence time $T_{2}^{*}$. The polarized ${ }^{3} \mathrm{He}$ can also be used as a comagnetometer to measure changes in the magnetic field. SQUIDS will be used to independently monitor the precession 
of the ${ }^{3} \mathrm{He}$ magnetization; the neutron density will be too low compared to the ${ }^{3} \mathrm{He}$ to affect this signal. The SQUID response can then be used to implement a time-dependent correction for background magnetic field shifts.

An undesired shift in the neutron spin precession frequency arises due to the coupling of magnetic field gradients and the motional magnetic field seen by the neutron motion in an electric field, $\overrightarrow{B_{m}}=\vec{v} \times \vec{E} / c^{2}$. This frequency shift is linearly dependent on the electric field, $E$, so it will appear as a "false" EDM term, $d_{f}$, in Equation 1 .

$$
\omega_{n}=-2\left(\mu_{n} B_{0} \pm\left(d_{n}+d_{f}\right) E\right) / \hbar
$$

This shift $\delta \omega_{n}$ in $\omega_{n}$ due to $d_{f}$ is predicted for a rectangular cell with a linear magnetic gradient by [15]:

$$
\delta \omega_{n}\left(\omega_{0}\right)=-\gamma^{2} \frac{E}{c^{2}}\left(\omega_{0} \operatorname{Im}\left[G_{y} S_{y y}\left(\omega_{0}\right)+G_{z} S_{z z}\left(\omega_{0}\right)\right]+G_{y} \frac{L_{y}^{2}}{12}+G_{z} \frac{L_{z}^{2}}{12}\right),
$$

where $\gamma$ is the gyromagnetic ratio of the neutron, $\omega_{0}=\gamma B_{0}$ is the precession frequency of the neutrons under the holding field, $S_{y y}$ and $S_{z z}$ are the spectra of the position correlation functions found in reference [16], $G_{y}$ and $G_{z}$ are the linear magnetic field gradients, and $c$ is the speed of light. The coordinate system is defined such that the magnetic holding field is in the $x$-direction; the $y$ - and $z$-directions are perpendicular to $x$, with $z$ along the axis of the cylindrical $\cos (\theta)$ magnet. $L_{y}$ and $L_{z}$ are the lengths of the cell in those directions. Given the geometry of the SNS nEDM design, we typically require linear gradients to be $G \leq 3 \times 10^{-6} B_{0} / \mathrm{cm}$, or a false EDM $d_{f} \leq 1 \times 10^{-28} \mathrm{e}-\mathrm{cm}$.

Novel techniques are needed to maintain magnetic uniformity in such a low-temperature environment. Use of magnetic components must be minimized, and those that are used must be placed as far from the cells as possible. The cells must be magnetically shielded. Additionally, temperature fluctuations in the cryogens are transferred to metal components, which generates magnetic fluctuations via Seebeck effect currents (thermoelectric effect). On the other hand, the cryogenic nature of the experiment offers the possibility of using a superconducting shield to exclude external fields via the Meissner effect.

\section{2. $1 / 2$-scale magnet prototype apparatus.}

As previously described in [1], a prototype magnet has been created at $1 / 2-$ scale of the nEDM at SNS magnet design in order to understand the level of magnetic uniformities achievable. That work described the field uniformity of the prototype magnet as measured at room temperature. We now describe a series of upgrades to that apparatus, including a helium cryostat and a lead shield, which when cooled below $7.2 \mathrm{~K}$ becomes a superconductor, shielding external magnetic fields.

A central magnet, referred to as the " $B_{0}$ " coil and seen in Figure 11 is used to produce a uniform magnetic field, typically $3 \mu \mathrm{T}$, using $\sim 50 \mathrm{~mA}$ current. The magnet follows a " $\cos (\theta)$ " design, which uses a cylindrical geometry with currents running on the surface parallel to the axis according to a cosine distribution. This design is known to generate throughout the cylinder a uniform field perpendicular to the axis of the coil [17].

The $B_{0}$ coil approximates the ideal $\cos (\theta)$ design using copper wires strung along a cylindrical surface with spacing determined by a modified cosine distribution, the details of which can be 
found in [1]. The coil used is $2.13 \mathrm{~m}$ tall, $0.61 \mathrm{~m}$ in diameter, and has 60 axial wires. The wires are wrapped on spring-tensioned pegs to maintain straightness. The pegs are mounted in acrylic rings, which are in turn supported by vertical rods made of alternating segments of G10 and nylon. The ratio of the lengths of the G10 and nylon segments is designed so that the thermal contraction of the entire rod will match the contraction of the copper wire. Finally, the entire structure is supported on a cylindrical acrylic frame, which has vertical grooves allowing the acrylic rings to slide.

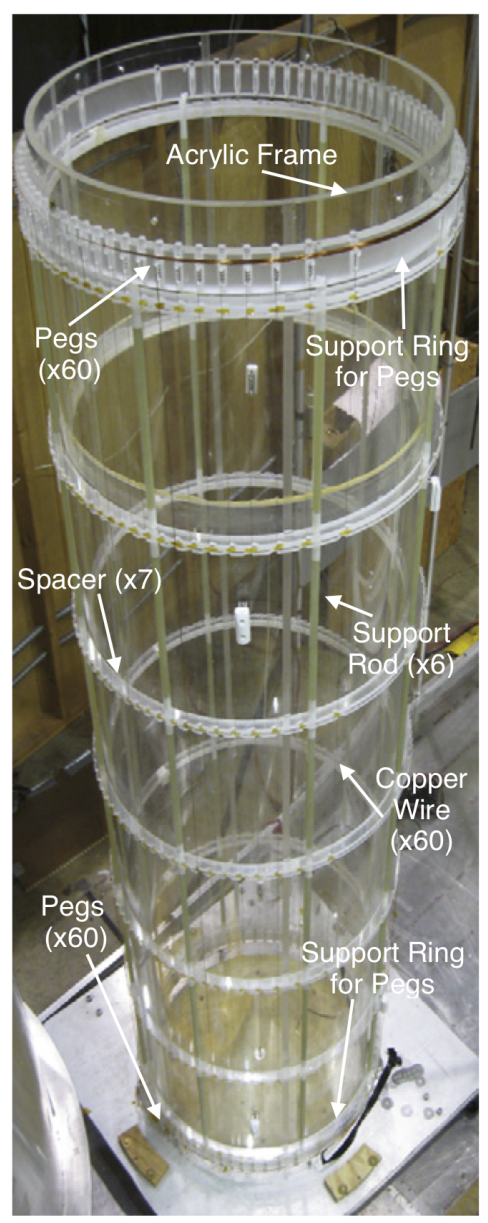

Figure 1: The $B_{0}$ coil. Copper wires are supported by pegs on springs at either end; the pegs and springs are mounted in acrylic. The frame is supported by the green/white G10/nylon rods, and seven acrylic rings have notches to keep the wires straight along the length of the cylinder.

Trim coils are used to compensate for field non-uniformities arising from local magnetic impurities or from the fact that the coil is discrete and not an ideal $\cos (\theta)$ design. It was found that three pairs of trim coils were necessary to cancel ambient gradients, as shown schematically in Figure 2 a $\cos (\theta)$ coil with 12 wires to trim the field in the $B_{0}$ direction; a rectangular coil with 


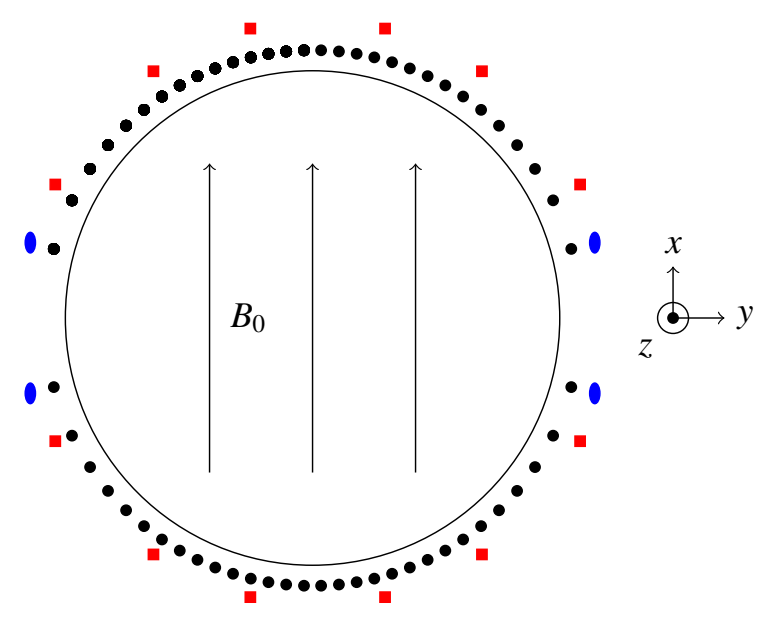

Figure 2: Bird's-eye-view schematic of $B_{0}$ and trim wire arrangements. Black dots and red squares indicate the $B_{0}$ wires and trim coil, respectively. Blue ellipses indicate the square quadratic trim coil.

5 turns to trim quadratic fields in the $B_{0}$ direction; and circumferential coils around the $B_{0}$ frame to control the field in the vertical direction. Each coil was split about its plane of symmetry, so that the current in each half could be controlled separately. This allowed for switching between a mode of maximum uniformity (for field magnitude control) or a gradient control mode. The trim coils are controlled by LabVIEW and powered by a Measurement Computing 16-bit digital to analog converter (DAC) (USB-3114) with $\pm 40 \mathrm{~mA}$ current capability per channel [18, 19].

The magnet system includes magnetic shielding to improve uniformity. To accommodate the cryogenic superconductor, the package of magnetic shielding and coils is operated inside a cryostat, discussed below. The entire apparatus is shown schematically in Figure 3 The cryostat itself is wrapped in four layers of Metglas 2705M and one layer of mu-metal to reduce the size of environmental fields, primarily the Earth's field. The Earth's unshielded magnetic field is of magnitude $\sim 50 \mu \mathrm{T}$ at the location of the cryostat, primarily in the vertical direction, along the axis of the apparatus.

Inside the cryostat, the outermost layer of the magnet package is a cylindrical superconducting lead shield, shown in Figure 4. The lead is $0.75 \mathrm{~m}$ in diameter, $2.2 \mathrm{~m}$ long, and $0.8 \mathrm{~mm}$ thick, and it is supported by a G10 fiberglass frame. G10 is chosen for its rigidity and its low thermal contraction coefficient. Copper pipes soldered to the lead and taped to the frame carry liquid helium to cool the shield. An ideal superconducting shield would expel all external magnetic flux via the Meissner effect, but in practice, magnetic flux can be trapped in impurities or inhomogeneities in the lead as it transitions below the superconducting transition temperature, $T_{C}=7.2 \mathrm{~K}$. Thus, it is important to minimize the magnetic fields at the surface of the lead before cooling it below the superconducting transition. Below $T_{C}$, the lead is very effective at mitigating time-varying external fields.

The $B_{0}$ field is perpendicular to the axial lead shield, but a superconductor admits no perpendicular magnetic field at its surface. Thus, there is a field distortion at the lead, and it is necessary to provide a flux return with the correct boundary conditions. To this end, a ferromagnetic (FM) shield was placed inside the lead shield, consisting of six layers of Metglas 2705M [20], wrapped 


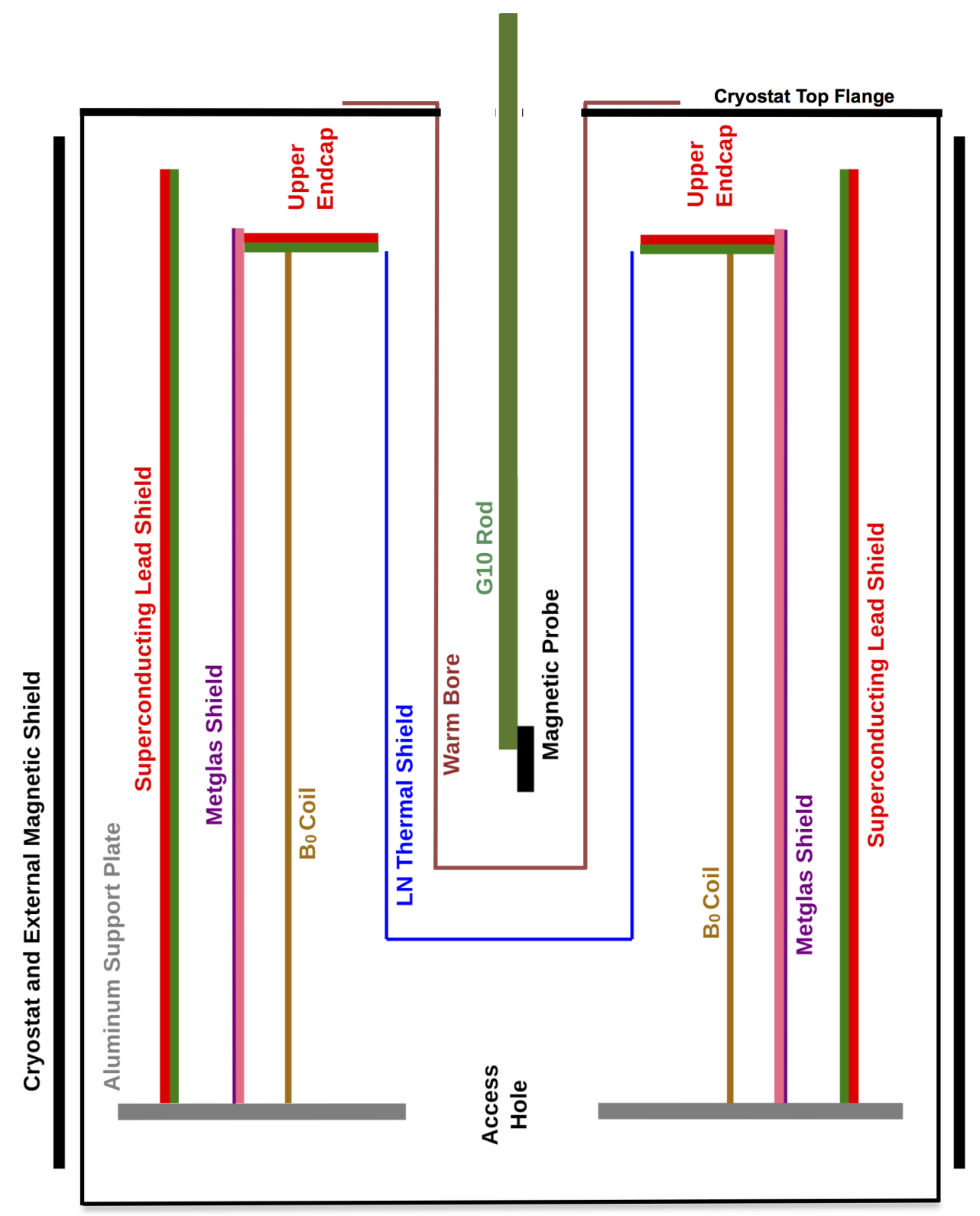

Figure 3: A schematic showing the nested arrangement of coils and magnetic and thermal shielding. Additional thermal shielding between the cryostat top flange and the rest of the components is not shown. Not to scale. 
$2.25 \mathrm{~m}$ in height along a $0.72 \mathrm{~m}$ diameter Vylon tube [21]. The layers are composed of strips 22 $\mu \mathrm{m}$ thick and $5.08 \mathrm{~cm}$ wide, with alternate longitudinal and circumferential layers to allow for magnetic flux continuity in both directions along the cylindrical surface. Metglas 2705M was chosen as it retains high permeability from room temperature to cryogenic temperatures. The FM shield also provides magnetic shielding [22] from environmental fields.

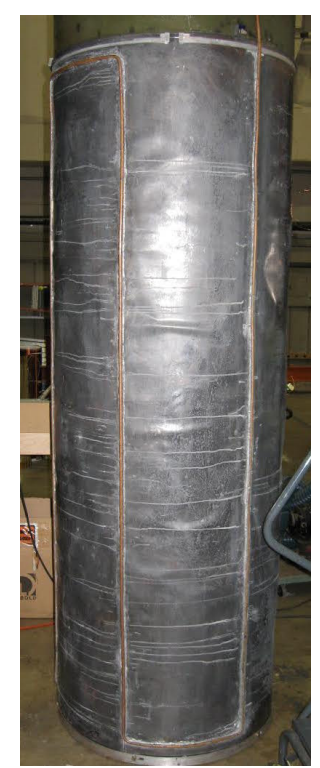

Figure 4: Lead shield used for superconducting magnetic shielding. The lead, $2.13 \mathrm{~m}$ high, is mounted on a G10 cylinder. Soldered copper cooling lines allow for thermal contact with liquid helium.

The mu-metal/Metglas external shield and the internal FM shield both include degaussing coils. The coils consist of 20 turns of $0.81 \mathrm{~mm}$ wire wrapped along the inner and outer surfaces of the shield, parallel to the axis. The loops penetrate both faces of each support cylinder, so as to form a segment of a toroid around each shield. The coils are powered by a California Instruments 801RP power supply, controlled via LabVIEW [23]. The degaussing current is a $60 \mathrm{~Hz}$ sinusoid modulated to ramp from zero amplitude to a peak of 5.5 Amperes, and then drop to zero again, in $\sim 5$ minutes. The external and internal shields are degaussed sequentially, in that order.

We noticed that the 801RP supply produced a small burst of uncontrolled current at the end of the ramp down, leaving a small field trapped in the Metglas. To eliminate this, we installed a transformer between the supply and the coils to isolate the coils. We also installed a LabVIEWcontrolled relay between the coils and the power supply which opens the circuit just before the burst occurs. A circuit diagram for this relay system is shown in Figure 5.

To further mitigate the flux trapped in the lead shield due to the Earth's field, rectangular compensation coils are installed in all three directions surrounding the magnetic testing area; see Figure 6 The vertical coils are $6.1 \mathrm{~m} \times 6.1 \mathrm{~m}$ with $3.7 \mathrm{~m}$ separation, while the $\mathrm{x}$-coils and y-coils are $6.1 \mathrm{~m} \times 3.7 \mathrm{~m}$ with $6.1 \mathrm{~m}$ separation. Each direction has a main and trim coil, respectively to adjust magnitude and gradients. Additional coils are installed in the $\mathrm{x}$-direction that bisect the main coil, effectively forming four equal coils of half the height and width; this provides finer 


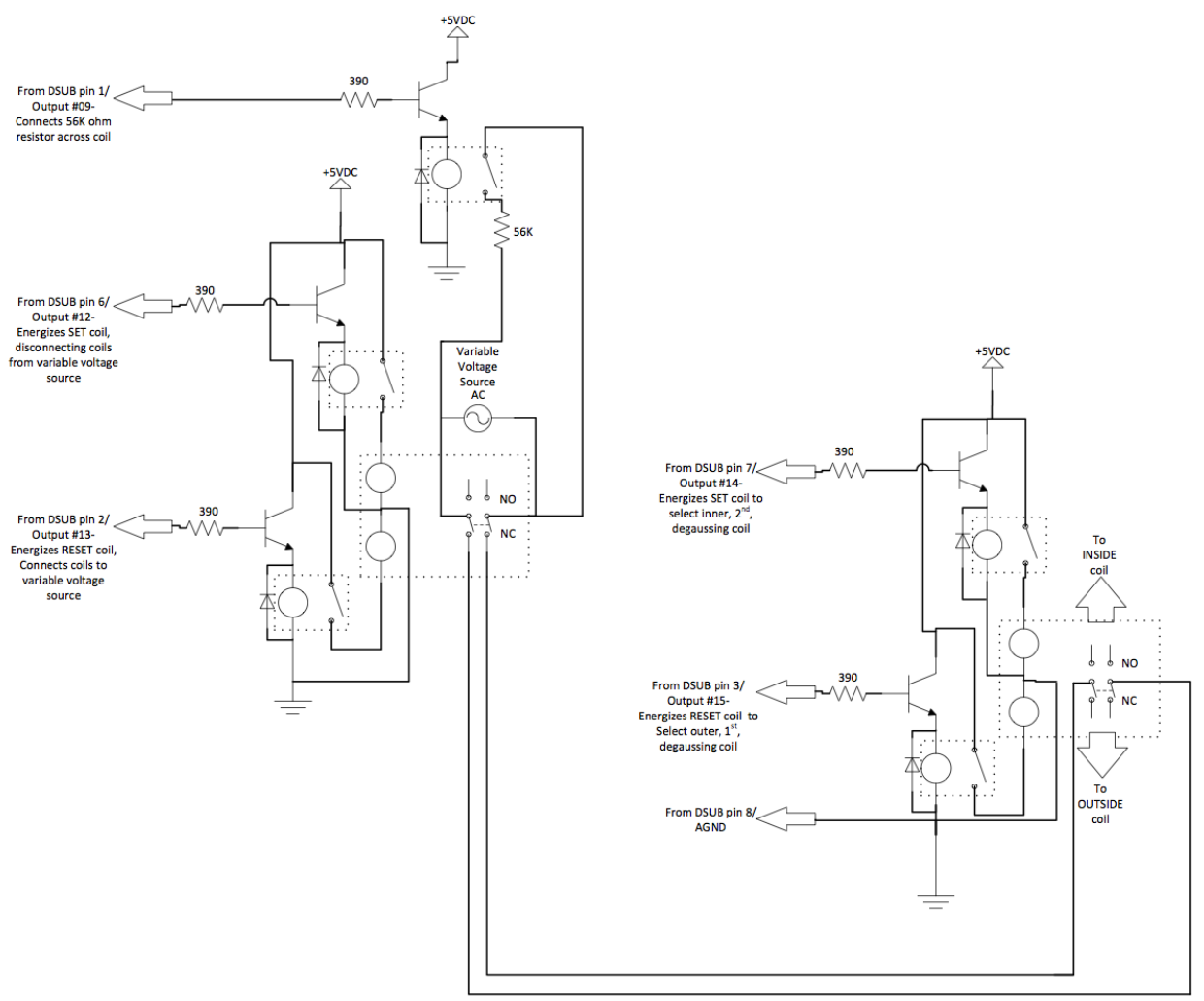

Figure 5: Circuit used to control mu-metal and Metglas degaussing. A 5.5 A current sweep is provided by the variable voltage source. One switch decouples the source from the degauss coils to protect the coils from noisy bursts of current at the end of the sweep. Another switch alternates between degaussing the external and internal magnetic shields. 
gradient control.

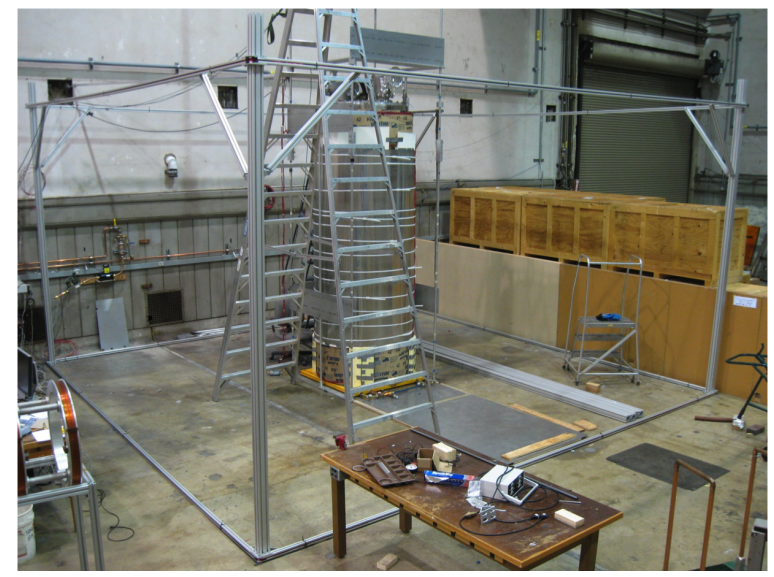

Figure 6: Ambient field compensation coils are wound on the metal bars shown surrounding the cryostat. Additional fine gradient coils are not shown.

The magnetic field is measured with a 3-axis, low-noise fluxgate magnetometer (Bartington Mag-03) [24]. The magnetometer signal is read out with a Signal Conditioning Unit (Bartington SCU1) and passed to a LabVIEW monitoring system via a 24-bit analog to digital converter (ADC) (Measurement Computing USB-2408). For automated field mapping, the probe is coupled to a stepper motor via a $3 \mathrm{~m}$ long G10 rod. The motor sits on top of an aluminum stand, placing any magnetic fields it produces about $3 \mathrm{~m}$ from the field mapping region. A 3-axis National Instruments motion controller (MID-7604) allows LabVIEW control of the motor [25].

\subsection{Superconducting endcap}

As part of experimental design optimization, it is of interest for the SNS nEDM experiment to explore the possibility of closing the ends of the superconducting lead shield. This would provide improved uniformity of the $B_{0}$ field, as well as additional shielding from external magnetic fields. Fringing fields from the open ends would also be reduced, so designs with more compact magnets and shields would become available, reducing engineering costs and challenges. To allow assembly of internal components, the ends would have to be removable; thus, a lead disk, or "endcap", is required to cover the ends of the cylindrical portion of the lead shield and form a nearly hermetic shield.

We created such a removable lead disk to fit inside the FM shield, roughly at the height of the top of the lead shield; see Figures 3 and 7. To accommodate the warm bore, the disk is annular, with OD $66 \mathrm{~cm}$ and ID $43.2 \mathrm{~cm}$. It sits on a G10 disk for support, which rests on top of the $B_{0}$ magnet frame. The lead has independent soldered-copper cooling lines, and the entrance to the lines was wrapped with heater wire to allow the superconducting state to be controlled for comparison.

There are significant limitations to this endcap arrangement. Only an upper endcap was installed; a lower endcap would have required modifications of the lower aluminum mount. This, combined with the central hole in the endcap, means the lead shield is not completely hermetic in this arrangement. Furthermore, the length of the lead shield means that the endcap is too 


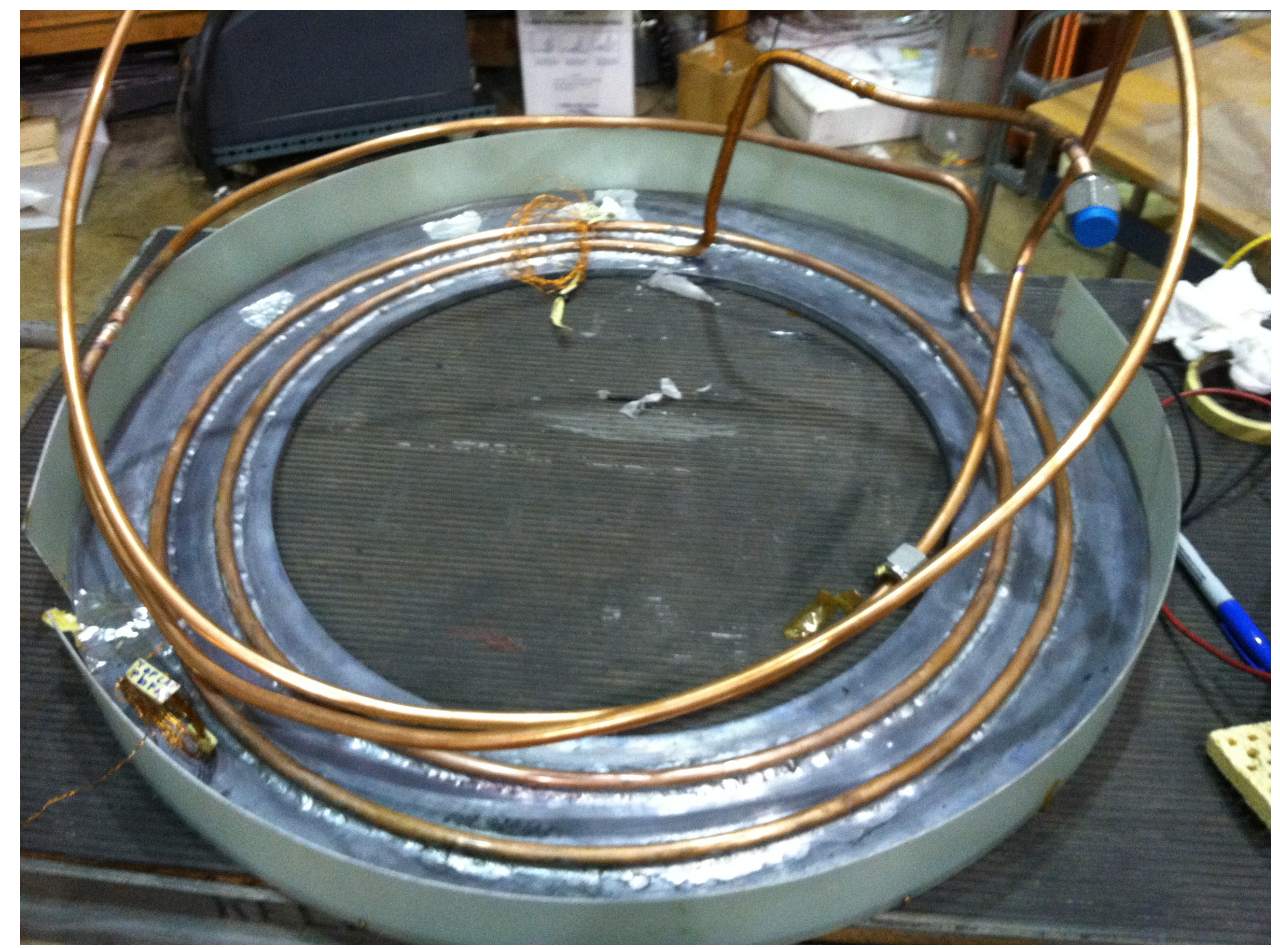

Figure 7: Superconducting lead endcap used to partially close an open end of the superconducting shield. The lead rests on a G10 frame (not seen) and has a G10 wall to retain its position. Copper tubing soldered to the lead provides liquid helium cooling. 
far away to measurably affect the field in the region of interest inside the $B_{0}$ coil. Thus, this prototype cannot be used to assess the endcap's effect on magnetic gradients; this will instead be explored in the future. Nevertheless, this prototype can be used to validate simulations of endcap designs, which improves the reliability of simulations for future designs. Even with a central hole, simulations of the annular endcap design predict a measurable improvement on field uniformity in the vicinity of the endcap. These results also are presently being used to further optimize the magnet package for the experiment.

\subsection{Cryogenic Apparatus}

The package of $B_{0}$ magnet and shields is operated in a $3 \mathrm{~m}$ tall, $1 \mathrm{~m}$ diameter triple-walled stainless-steel cryostat. The cryostat is liquid nitrogen and liquid helium cooled, and contains bayonet penetrations for liquid helium service to the package. The lid contains a deep indent in the center, a "warm bore" $\sim 1.5 \mathrm{~m}$ deep and $0.28 \mathrm{~m}$ diameter, to allow a room-temperature probe to access to the interior of the $B_{0}$ coil.

Inside the cryostat, the magnet package is supported from below by a plate of 1100-alloy aluminum, which is suspended from the cryostat lid by G10 rods. This alloy was chosen for having a larger thermal conductivity than other aluminum alloys at cryogenic temperatures. The plate is helium cooled, providing additional thermal shielding and conductive cooling. A helium-cooled aluminum radiation shield ("mushroom") is placed on top of the magnet package, covering the top of the package and part of its side. The mushroom has a cut-out in the top for the warm bore to pass through. Both the aluminum plate and the mushroom have 3003-alloy aluminum cooling lines attached via dip-brazing [26] 1 The aluminum cooling lines are coupled to stainless-steel

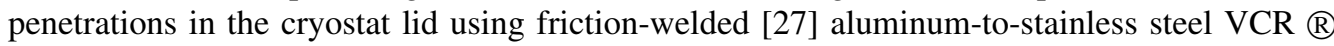
connectors [28]. The room-temperature bore is surrounded by an aluminum thermal shield 0.33 $\mathrm{m}$ in diameter, which is cooled via copper tubes taped to the sides. The warm bore and radiation shields are covered in 10-layer multi-layer insulation (MLI) to improve thermal shielding.

\subsection{Cryogenic Design}

Electrical currents can be generated by thermal gradients in the metal components via the Seebeck effect. These currents produce stray magnetic fields that can add magnetic noise and non-uniformities in the measurement region. Aluminum was preferred for metal parts inside the cryostat since it has a lower Seebeck coefficient than other natural choices, such as copper. Still, due to the presence of some copper lines, magnetic fluctuations correlated with temperature gradients were observed. Hence, it was necessary to implement measures to stabilize the temperatures in the liquid cryogen.

A schematic of the cryogenic flow system is shown in Figure 8, Liquid nitrogen and helium supply dewars are pressurized with the respective gas to increase flow and ensure uniformity of the supply pressure. The pressure is supplied through regulators which are controlled using LabVIEW via a 16-bit digital to analog converter (DAC) (Measurement Computing USC-3102). Mass flow controllers (Alicat MCR series, [29]) on the cryogen exhaust dramatically improve the temperature and magnetic stability of the system. The controller for the helium is modified, at our request of the manufacturer, with an option to control on either pressure or mass flow as

\footnotetext{
${ }^{1}$ Initially, the plate and mushroom were copper-plated and had copper cooling lines attached by brazing. However, the plating process included nickel, leaving measurable magnetization. The copper plating and copper tubing were removed by abrasion and chemical processes before attaching the aluminum cooling lines.
} 
the process variable. Pressure control was found to further increase temperature stability. The flow controller parameters are set using Labview via serial connections.

Pressure and temperatures sensors are located in key places in the system and monitored with a Labview-based "slow-control" program, which is the same program that controls the cryogen regulators and flow controllers. Pressure data is read in using a 12-bit National Instruments ADC (USB-6008). Temperature data is acquired from a mixture of $\sim 30$ platinum resistance temperature detectors and $\mathrm{Si}$ diodes, respectively for liquid-nitrogen and liquid-helium cooled areas of the system. These signals are read and calibrated using control units (Lakeshore 218 [30]), and from there are passed into slow-control via serial connection.

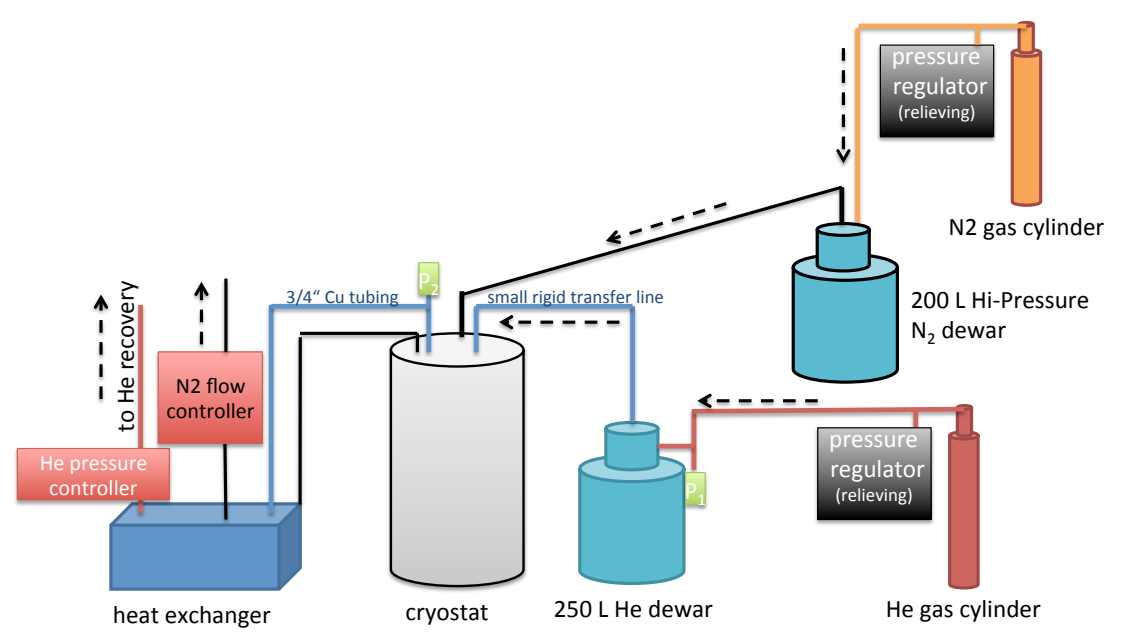

Figure 8: A schematic of liquid cryogen control system. Gas cylinders pressurize liquid cryogens, and the flow rates are regulated at the output. A heat exchanger protects the flow-controllers from low temperatures.

\section{Results}

\subsection{Thermal Stability}

Fluctuations in cryogenic component temperatures were observed to correlate strongly with magnetic fluctuations in both nitrogen-cooled and helium-cooled components. Figure 9 shows this correlation for the temperature of the warm bore liquid nitrogen thermal shielding. Here the mass flow rate of the nitrogen vapor is controlled. To mitigate these fluctuations in liquid nitrogen, the flow was simply shut off during sensitive magnetic measurements. Temperatures in the nitrogen-cooled components were allowed to drift upward, which occurred at a rate of 1 $\mathrm{K} / \mathrm{hr}$, too slowly to induce noticeable magnetic changes.

The corresponding correlations between the fluctuations in the liquid helium temperature and measured magnetic field are shown in Figures 10 and 11 for mass flow control. A 0.032 


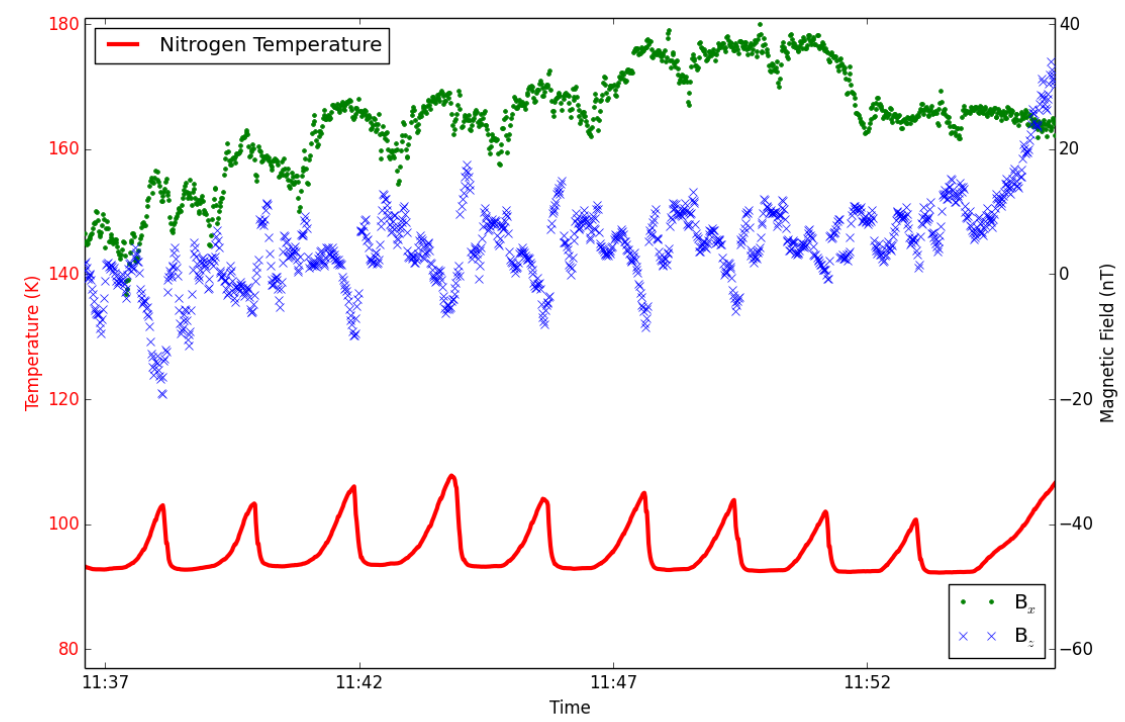

Figure 9: Magnetic field fluctuations correlated with temperature fluctuations in the liquidnitrogen-cooled portions of the thermal shielding. $B_{z}$ oscillates in phase with the temperature, while $B_{x}$ oscillates with the same period, but out of phase. The liquid nitrogen mass flow rate is regulated. Stopping the liquid-nitrogen flow reduced the magnetic fluctuations to negligible levels (not shown). 


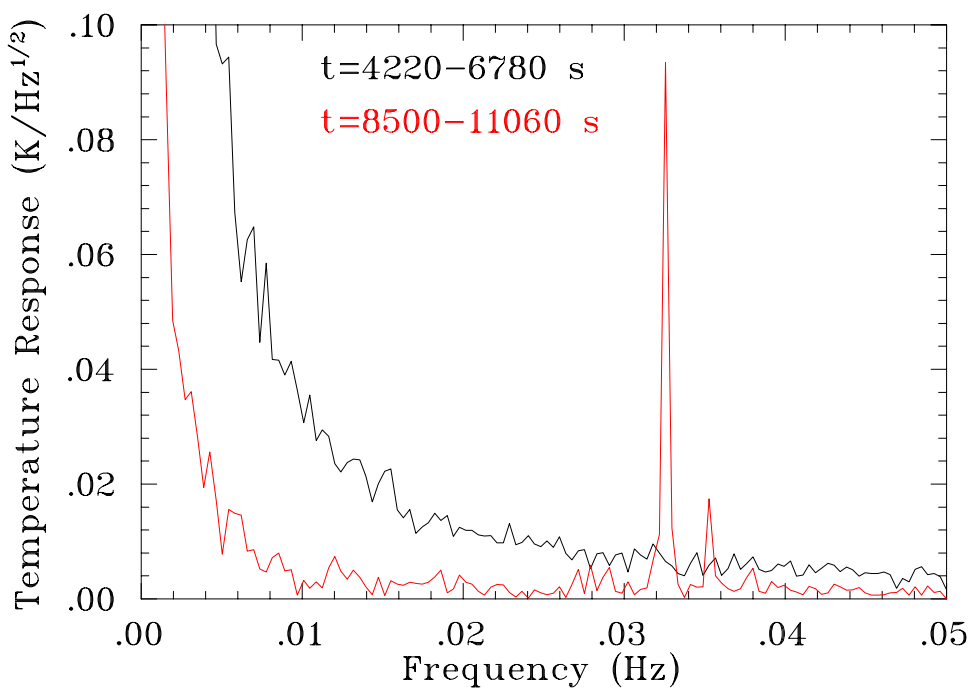

Figure 10: Fourier transform of the time-dependence of the lead superconductor temperature, with liquid-helium flow controlled based on output pressure (black) and mass flow rate (red). Pressure control eliminates the strong characteristic thermal fluctuation at $0.032 \mathrm{~Hz}$. The time intervals in the legend are sequential; the control mode was changed in the intervening time to demonstrate the effect. Note that the liquid-nitrogen flow was stopped for these measurements. 


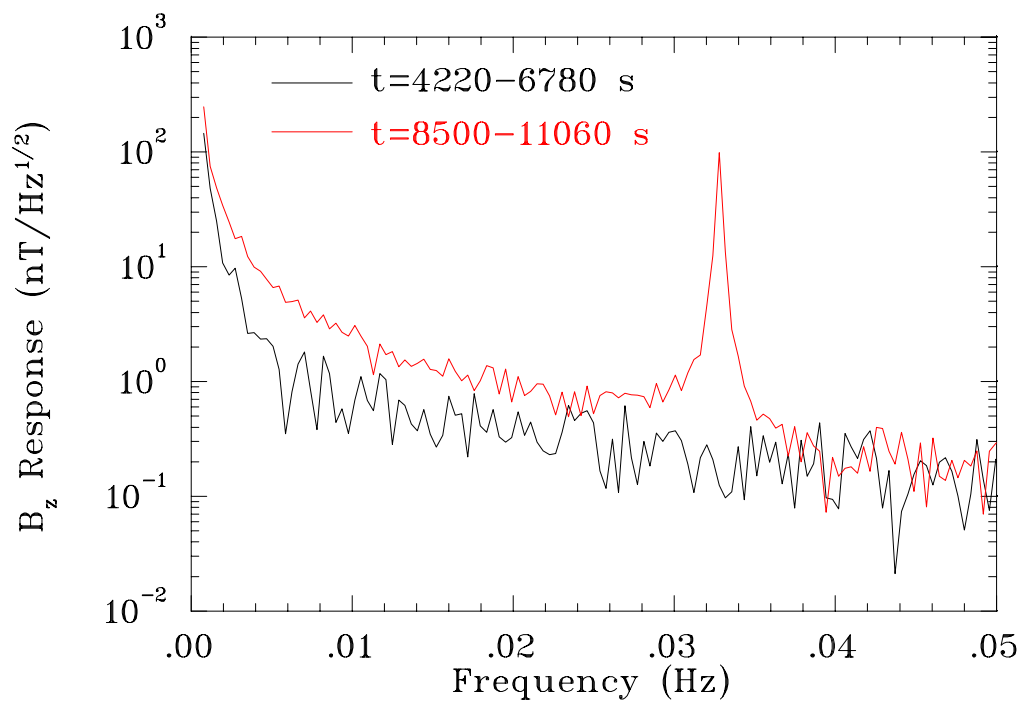

Figure 11: Fourier transform of the time-dependence of the vertical component of the magnetic field measured at the center of the $B_{0}$ magnet. The time intervals correspond to Figure 10 , with liquid helium flow controlled based on output pressure (black) and mass flow rate (red). The magnetic field suffers a fluctuation precisely corresponding to that of the temperature when in mass-flow-control mode, and is correspondingly eliminated in pressure-control mode. Note that the liquid-nitrogen flow was stopped for these measurements. 


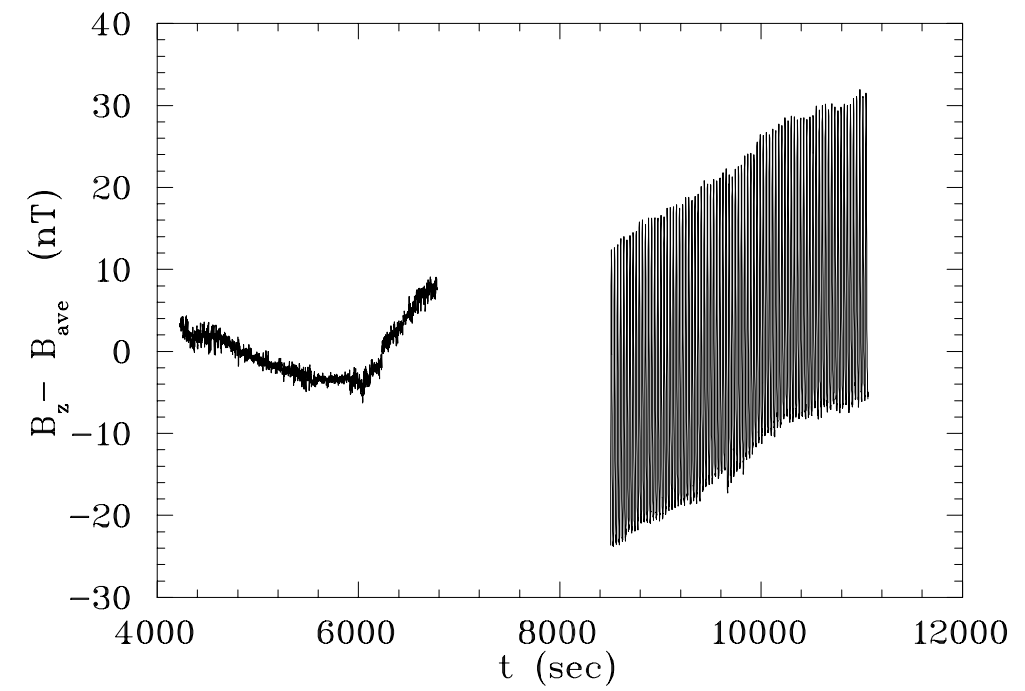

Figure 12: Deviation of $B_{z}$ from average, with liquid helium flow pressure-controlled before $8000 \mathrm{~s}$ and mass-flow-controlled after $8000 \mathrm{~s}$. The control mode was changed to demonstrate the effect. 
$\mathrm{Hz}$ characteristic temperature oscillation frequency is clearly present when the helium mass flow rate is controlled. For precise measurements of the magnetic field it was necessary to eliminate this oscillation, and shutting off the helium flow was not possible as the superconducting lead would rise above the critical temperature too quickly. In order to reduce the thermal fluctuations, we changed from mass flow control to vapor pressure control by replacing the liquid helium mass flow controller with a similar device from the same company capable of regulating the exhaust helium pressure as well. By tuning the PID feedback parameters of the controller, the fluctuations in both temperature and magnetic field could be reduced below the sensitivity of our measurements, as seen in the black curves in Figures 10 and 11 The effect is also clearly visible in the time dependence of the magnetic field for the case of pressure control (early times) and mass flow control (late times) as seen in Figure 12 .

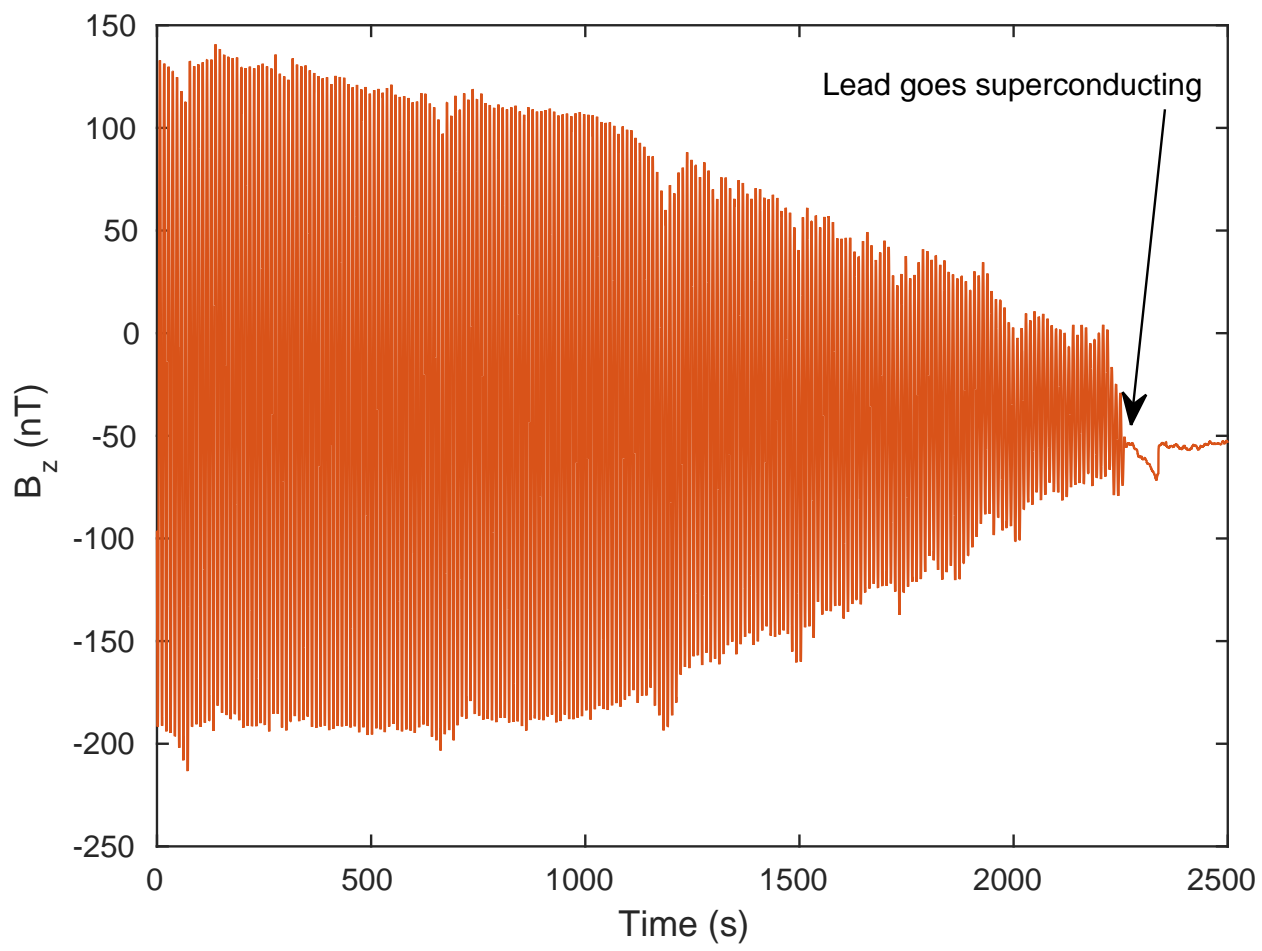

Figure 13: The reduction of noise, as simulated by a $0.1 \mathrm{~Hz}$ quasi-static AC magnetic field, as the lead transitions into the superconducting state. 


\subsection{Magnetic Shielding}

The superconducting shield is effective at mitigating the effects of transient changes in the external magnetic environment. Figure 13 shows a visualization of the transition of the lead shield to the superconducting state. A $0.1 \mathrm{~Hz}$ quasi-static AC signal is generated in the axial compensation coils as a simulation of a noisy environment, and the field is monitored by the probe at the center of the lead shield as it goes from the normal state to superconducting. After the transition a dramatic reduction in the "noise" is observed.

To quantitatively characterize the effectiveness of the shield, we apply a quasi-static AC driving field using the compensation coils outside the cryostat. The AC current driving the field is made large enough to be measured with the lead shield in the superconducting state; this is observed to be $B_{\text {unshielded }}=1.44 \mu \mathrm{T}$ in amplitude at the probe position in the absence of shielding for all frequencies. We measure the field at the center of the shield, then measure the field at the same location after the shield is removed. We can then define a shielding factor, $S$, as the ratio of the two measurements:

$$
S=\frac{B_{\text {unshielded }}}{B_{\text {shielded }}} .
$$

Induced eddy currents and skin depth effects lead to a frequency dependence in the shielding factor, providing enhanced shielding of magnetic fields at higher frequencies. Thus, the quasistatic shielding factor is determined from the asymptotic behavior of the shielding factor at low frequencies. Using Equation 4 , we measure a total axial shielding factor $S^{A}=4882 \pm 174$. The total DC transverse shielding factor is $S^{T}=79.0 \pm 3.1$. Uncertainty in the axial or transverse total shielding factor is determined from the magnitude of the background noise spectrum at the driving frequency.

We are also interested in extracting shielding factors due to the superconducting shield itself, $S_{S C}$, as opposed to the total shielding factor, $S$, which includes other metallic and ferromagnetic components. In order to do this we measure the magnetic field - $B_{N}$ - with the lead shield in the normal state above the superconducting transition temperature and - $B_{S C}$ - with the shield in the superconducting state below the transition temperature. Because of its finite conductivity above the transition temperature, the lead also can shield magnetic fields due to induced eddy currents and skin depth effects. Again, these effects are only important at higher frequencies. Thus, assuming that the various components of the shielding (ferromagnetic and conductor effects) enter multiplicatively, we can extrapolate $B_{N} / B_{S C}$ to low frequency in order to determine the superconducting shielding factor for the lead.

However, the analysis is complicated by several factors. First, since we are comparing results at two different temperatures, we want to insure that most of the other shielding is not changed for the two measurements. Thus, we don't want the temperatures to differ by an amount that will cause a significant change in the conductivity, and hence skin depth, of the components beyond the lead. To that end, we performed the $B_{N}$ measurements with the lead at $T \lesssim 30 \mathrm{~K}$. Another complication was the appearance of additional magnetic fields from eddy currents in the conductors near the magnetometer (e.g., the warm bore and LN shield). At higher frequencies this can actually increase the observed magnetic field, especially for axial measurements, due to local oscillating dipoles from the induced currents that are not shielded to the extent of the nearly uniform fields produced by the external coils. However, since this effect should show up for both $B_{N}$ and $B_{S C}$ measurements it should largely cancel in the ratio of the fields. 
In order to perform this extrapolation, we model the shielding of the normal state lead due to the induced eddy currents. It turns out that this effect dominates over the skin depth effect for the lead due to the large radius $(R=0.38 \mathrm{~m})$ and small wall thickness $(d=0.8 \mathrm{~mm})$ - this effect is discussed in Ref. [31]. To model this effect we consider the oscillating magnetic field as a source of EMF the drives a series $L R$ circuit, which is the lead conducting cylinder. In the axial case the EMF induces currents circulating azimuthally around the cylinder. For a long cylinder (length $\ell \gg R$ ) the fields can be calculated analytically as follows.

Assuming an applied field of $H_{0}$ varying harmonically as $e^{i \omega t}$, a solenoidal current $I_{s}$ will be induced which produces a net interior magnetic field of $B_{i}=B_{0}+B_{s}$, where $H_{s}$ is the field resulting from $I_{s}$. The EMF is given by Faraday's law

$$
\mathcal{E}=-\frac{d \Phi}{d t}=-i \omega B_{0}\left(\pi R^{2}\right)=I_{s} Z
$$

where $Z$ is the total impedence $Z=Z_{R}+Z_{L}$.

For the axial geometry with $\ell \gg R$ we have $Z_{L}=i \omega \mu_{0} \pi R^{2} / \ell$ and $Z_{R}=2 \pi R / \sigma d \ell$. This case is a solenoidal current distribution so that the field from the shield is uniform with magnitude $B_{s}=\mu_{0} I_{s} / \ell$. This gives, using $B_{i}=B_{0}+B_{s}$, the complex ratio

$$
\frac{B_{i}}{B_{0}}=\frac{1}{1+\frac{i R d}{\delta^{2}}},
$$

where $\delta$ is the skin depth of the shield given by

$$
\delta=\left(\frac{2}{\sigma \mu_{0} \omega}\right)^{1 / 2},
$$

in agreement with the result of Fahy, Kittel, and Louie [31]. The sign difference in the denominator is due to our choice of phase. This then gives a reduction of the internal field of

$$
\frac{\left|B_{i}\right|}{\left|B_{0}\right|}=\frac{1}{\sqrt{1+\frac{R^{2} d^{2}}{\delta^{4}}}}
$$

For the transverse geometry with $\ell \gg R$ we have $Z_{L}=i \omega \mu_{0} \ell / 2$ and $Z_{R}=2 \ell / \sigma d \pi R$. In this case we have a saddle current distribution with a current distribution varying as $\cos \theta$ traveling along the length of the tube, so that again we have a uniform field from $I_{s}$ given by $B_{s}=\mu_{0} \ell / 2$, which gives

$$
\frac{\left|B_{i}\right|}{\left|B_{0}\right|}=\frac{1}{\sqrt{1+\frac{R^{2} d^{2}}{\delta^{4}}\left(\frac{\pi}{2}\right)^{2}}} .
$$

In both cases there are finite size effects that modify the geometrical factors, but the frequency dependence should be approximately the same. 
In order to extract the quasi-static superconducting shielding factor, a fitted curve of AC measurements is extrapolated to zero frequency. As the frequency tends to infinity, the shielding factor for the normal shield should approach that of the superconductor, as the boundary conditions are the same. In order to account for this, we approximate the normal state shielding factor as the harmonic mean of the superconducting shielding factor and the $\ell \gg R$ shielding factor calculated above

$$
\frac{1}{S_{N}}=\frac{1}{S_{S C}}+\left|\frac{B_{i}}{B_{0}}\right|,
$$

Then with $S_{N}=B_{P b} / B_{N}$ and $S_{S C}=B_{P b} / B_{S C}$ where $B_{P b}$ is the net field incident on the lead we obtain

$$
\left(\frac{B_{N}}{B_{S C}}\right)=1+\frac{S_{S C}}{\sqrt{1+\alpha_{k}^{2} \omega^{2}}}
$$

where the index $k$ can indicate either axial $\left(\alpha_{a}\right)$ or transverse $\left(\alpha_{t}\right)$ shielding. These values are given by

$$
\begin{gathered}
\alpha_{a}=\left(\frac{R d \sigma \mu_{0}}{2}\right)^{2} \\
\alpha_{t}=\left(\frac{\pi R d \sigma \mu_{0}}{4}\right)^{2} .
\end{gathered}
$$

The measurements, along with the best fits using equation 11 , are plotted in Figures 14 and Figure 15 for the axial and transverse cases respectively. The extracted shielding factors from the best fits are given in Table 1 along with the total shielding factors discussed earlier.

\begin{tabular}{ccc}
\hline Shielding Factors & Total $(S)$ & Superconducting Shield $\left(S_{S C}\right)$ \\
\hline Axial & $4882 \pm 174$ & $1183 \pm 131$ \\
Transverse & $79.0 \pm 3.1$ & $15.5 \pm 1.2$ \\
\hline
\end{tabular}

Table 1: Shielding factors for the entire apparatus and for the superconducting shield alone, as measured at the center of the apparatus with applied AC magnetic fields.

The fit to equation 11 for the transverse case implies a lead conductivity of $\sigma=6 \times 10^{8} \mathrm{~S} / \mathrm{m}$, which is consistent with high purity lead around $20 \mathrm{~K}$. The temperature of the lead during the "normal" shielding measurements was recorded as $32 \mathrm{~K}$ and $24 \mathrm{~K}$ on the center and bottom of the cylinder respectively. However, the fit for the axial case implies a much larger conductivity by nearly a factor of 20 . This corresponds to temperatures near the superconducting temperature. Since the lead is actively cooled with cold He lines soldered to the surface, it is possible that the circulating induced currents for the axial case sample lead at lower temperatures.

In order to help understand the response of the full system, a model of the apparatus was simulated in COMSOL Multiphysics ${ }^{\circledR}$ software [32] at higher frequencies. Due to the limitations in 


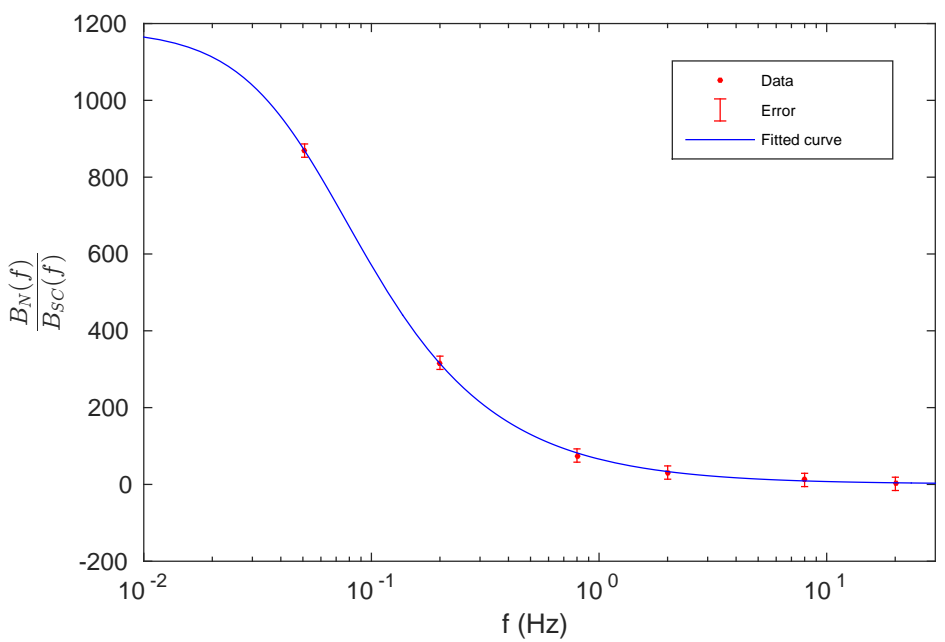

Figure 14: The axial superconducting shielding factor is extrapolated from measurements of the lead in superconducting and normal states. The DC value is extrapolated assuming a general skin depth formulation arising from the lead shield in the normal state at $T=33 \mathrm{~K}$. Error is dominated by the noise with the lead shield in the normal state.

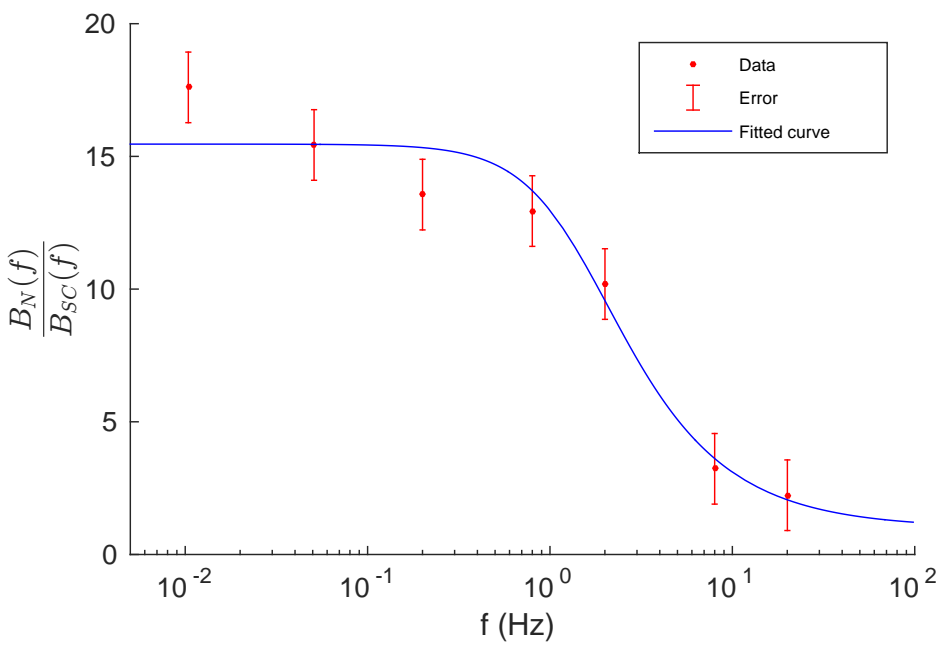

Figure 15: The transverse superconducting shielding factor is extrapolated from measurements of the lead in superconducting and normal states at $T \sim 30 \mathrm{~K}$. The DC value is extrapolated assuming a skin depth associated with the normal lead shield. Error is dominated by the noise with the lead shield in the normal state. 


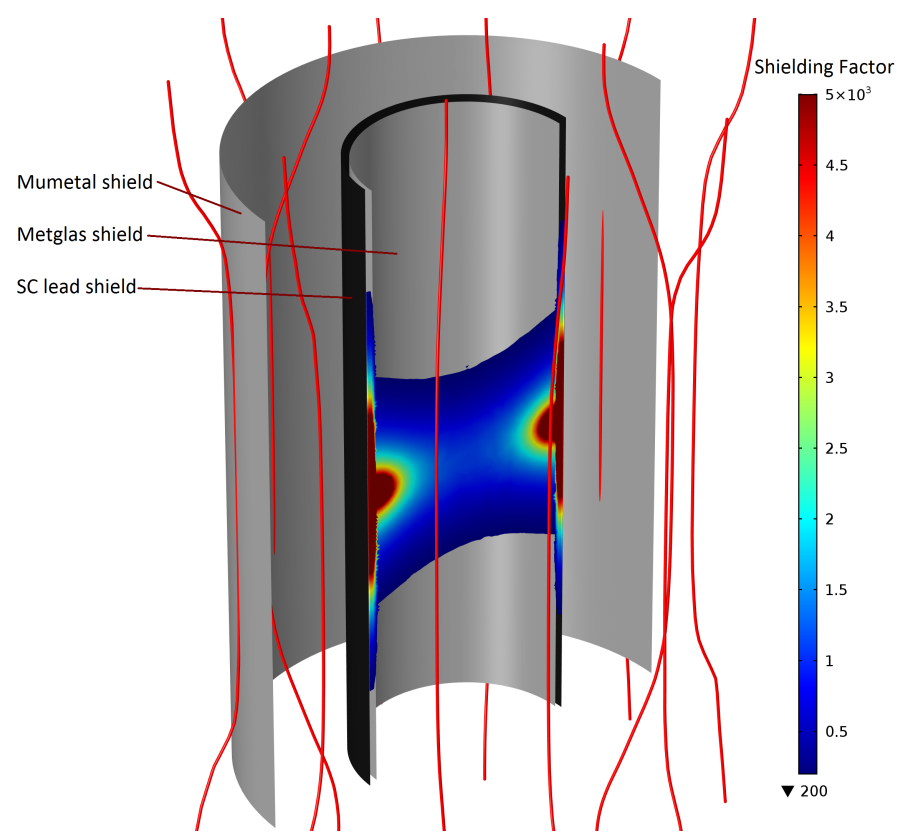

Figure 16: Visualization of the DC axial shielding factor simulated in COMSOL. Magnetic field lines are shown in red.

memory the geometry of the model is rudimentary: the warm bore and the warm bore's nitrogen shield are each separately simulated along with the cryostat top flange. Furthermore, smaller features are not simulated; for example the cooling lines on the nitrogen shield and the aluminized mylar used for insulation are larger features that are not included due to memory constraints.

The model predicts shielding factors of $S_{S C}^{A}=1108$ and $S_{S C}^{T}=19.4$, comparable to the measured values given in Table 1 Examples of the predicted field lines and local shielding factors of the model are shown in Figures 16 and 17

\subsection{Superconducting endcap results}

The effects of the superconducting endcap were predicted using magnetic simulation to solve for magnetic fields resulting from cylindrically symmetric shields comprised of both ferromagnetic and superconducting materials. The simulation solves for the Green's function with the provided boundary conditions, and proceeds to integrate the Green's function with respect to the requested current density and acquisition points. The calculation includes singular-value decomposition solvers allowing solutions with current densities that circulate on a global geometric scale, an ingredient required for superconducting shields. Small penetrations into the superconducting shield can be accounted for in the simulation by the application of a dipole field at the position of the penetration, with the strength of the dipole calculated according to [33].

The effects of the endcap are negligible at the center of the fiducial cell, due to the elongated geometry of the magnetic shielding, so it is necessary to map near the region of the endcap. This region is dominated by fringing fields which bow out the open end of the main cylindrical shield, leading to larger vertical $(z)$ magnetic field than other regions inside the cylinder. Simulation 


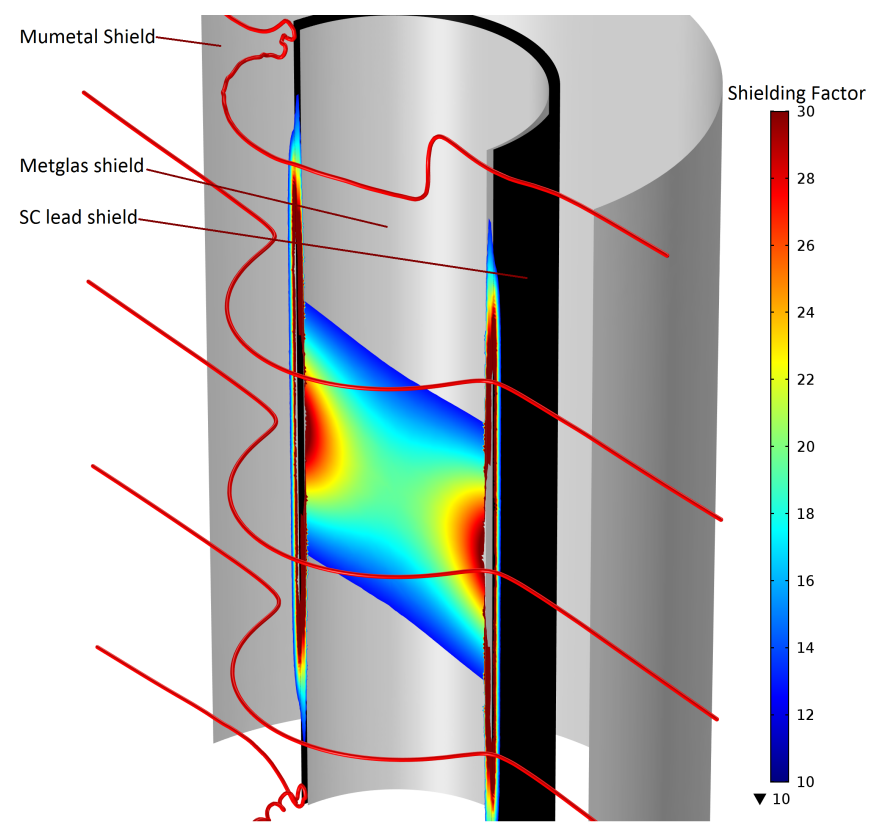

Figure 17: Visualization of the DC Transverse shielding factor due to the superconducting shield simulated in COMSOL. Magnetic field lines are shown in red.

shows that in the bulk of the lead-enclosed region, a superconducting endcap reduces the $z$-field, leading to more uniform $B_{0}$ field throughout the volume. However, in and above the hole at the center of the endcap, the $z$-field magnitude increases. In effect, the vertical magnetic field is "pushed out" of the lead region through the hole in the top.

Our magnetic probe can be used to map the magnetic field for both the normal and the superconducting state of the lead endcap. The vector magnetic field was sampled along a vertical slice passing from the center of the cell to $\sim 0.5 \mathrm{~m}$ above the endcap. The slice was chosen to be $0.104 \mathrm{~m}$ away from the axis, in the $B_{0}$ direction, since symmetry prevents any effect at points on the axis. Samples are taken in the presence of a nominal $B_{0}$ field. Measurements are shown in Figure 18 and compared to simulation, which is in excellent agreement.

\subsection{Magnetic Gradients}

Magnetic gradients are measured inside two rectangular fiducial regions in the center of the $B_{0}$ magnet, corresponding to the two measurements cells in the full $\mathrm{nEDM}$ experiment, but scaled down to half-size. The regions are $3.8 \mathrm{~cm} \times 5 \mathrm{~cm} \times 20 \mathrm{~cm}$ in extent, respectively along the $x$, $y$, and $z$ directions (see Figure 2); they are separated by $5 \mathrm{~cm}$ along the $B_{0}$ direction, and are referred to as the "left" and "right" measurement cells. Measurements are taken at night when man-made magnetic activity in the vicinity is reduced. Thermally-driven magnetic fluctuations are mitigated by tuning the liquid helium pressure control and by stopping the flow of liquid nitrogen, as described in Section 3.1. The endcap is not in use during maps; it is also too far removed from the cell to have significant effects even if it were superconducting. 
Field slice in [m]: $x=0.104, y=0.0$

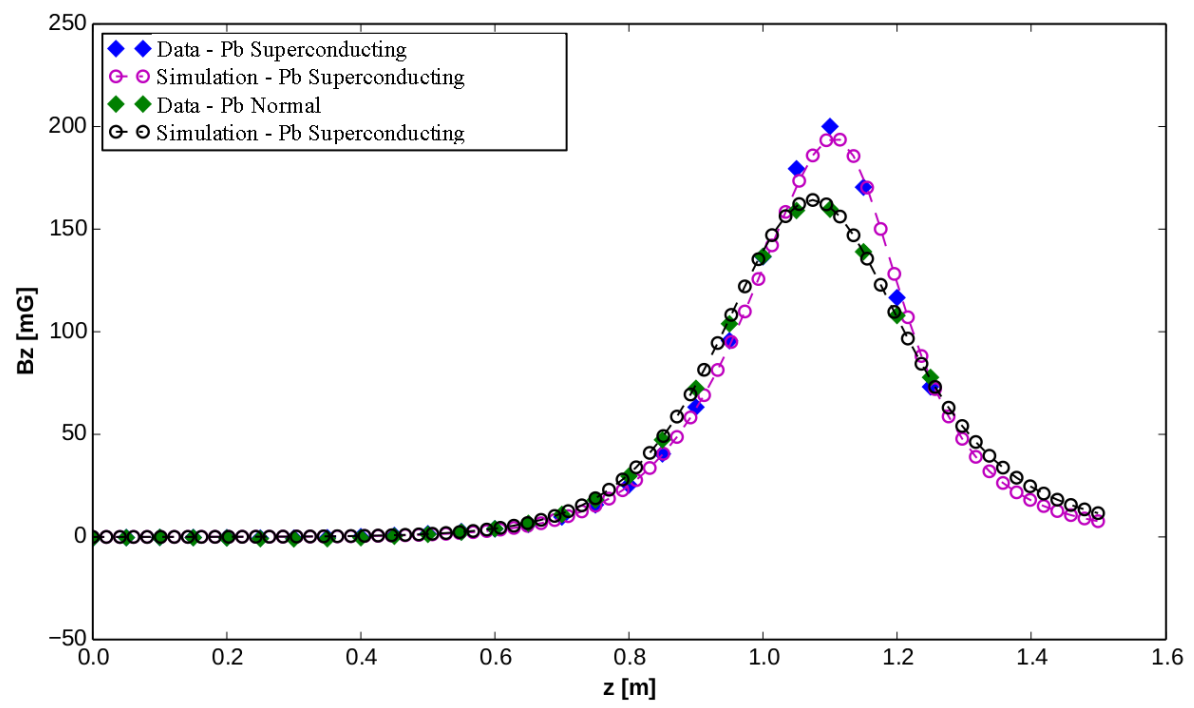

Figure 18: Simulated and measured vertical magnetic field along a vertical slice for superconducting (SC) and normal endcap. The slice is taken off-axis, $0.104 \mathrm{~m}$ in the $B_{0}$-direction. The vertical field peaks in the vicinity of the endcap; below the endcap the vertical field is smaller in magnitude when the endcap is SC, indicating greater field uniformity. 
A magnetic "map" is defined as a three-dimensional sampling of the fiducial region. The fluxgate magnetometer is moved from point to point along a pre-defined grid. The magnetic field is measured along 3 orthogonal axes at each point after a settling time of $1 \mathrm{~s}$ to reduce vibrational noise.

Trim coils are optimized to ambient conditions before taking a map. This is done iteratively in a series of test maps. A coarse map, lasting about 10 minutes and sampling tens of points, is taken. Trim coils are adjusted by hand to cancel any observed gradients.

Once trim coils are set, a detailed map of about 5 hours is taken with hundreds of points spaced about $1 \mathrm{~cm}$ apart. The map is fit using a 3-d quadratic polynomial for each field component. In the 3-d quadratic polynomial model the offsets and coefficients of all possible variations of the three dimensional Cartesian coordinate variables up to second order are fit simultaneously. Example fits are shown in Figure 19, along with fit residuals. Gradients are extracted from the linear components of the fit. Fit residuals of $\lesssim 1 \mathrm{nT}$, as compared to the measured field values of $\sim 30 \mu \mathrm{T}$, show that statistical uncertainties on the extracted gradients are negligible. The best gradients achieved are shown in Table 2, where they have been scaled to the nominal use $B_{0}$ field of $3 \mu \mathrm{T}$ and adjusted to the full-scale apparatus.

For this scaling we have assumed that the gradients are dominated by, and thus proportional to, the coil field, $B_{0}$ (noting that the observed gradients are much larger than the ambient gradients). The gradients then scale with the dimensions of the coil and are a factor of 2 smaller for the full-scale system. The values listed in Table 2 result in a geometric-phase-induced false dipole moment $d_{f}<1 \times 10^{-28} \mathrm{e}-\mathrm{cm}$.
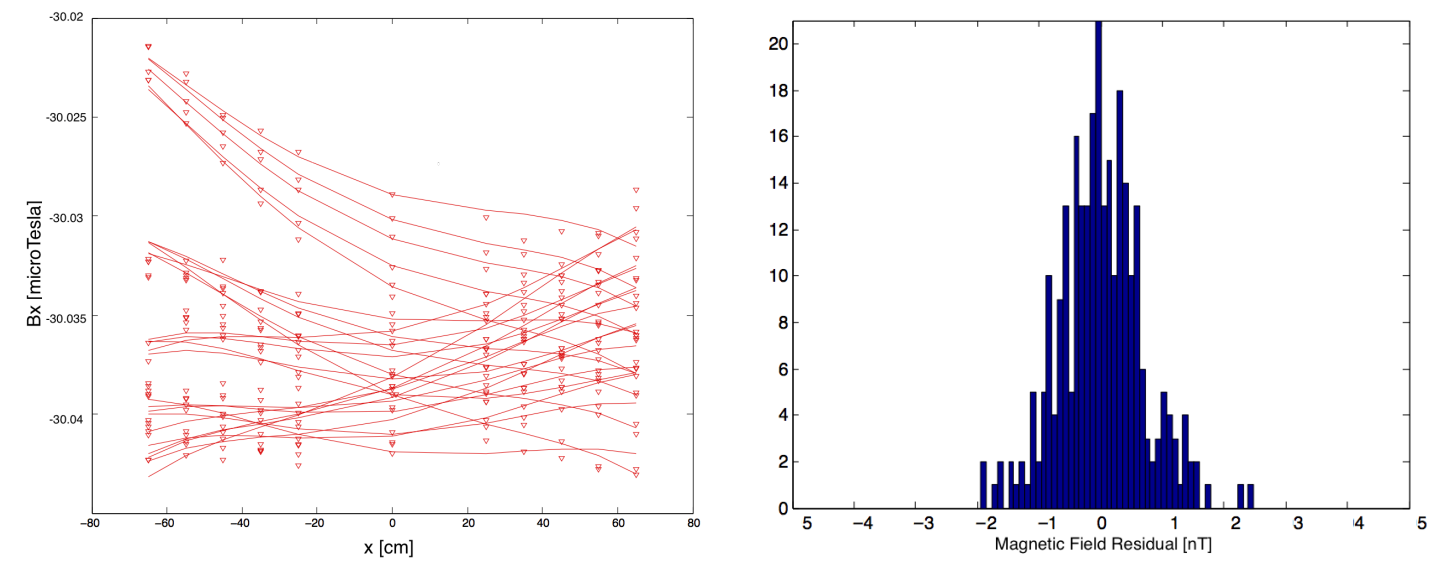

Figure 19: Fits (left) and residuals (right) for magnetic maps. A quadratic 3-d polynomial fit is used; the figure shows the projection of the fit to $B_{x}$ onto the x-direction. Note that the lead endcap is installed but not in the superconducting state for these measurements.

\section{Conclusions}

A prototype magnet system for the nEDM experiment at SNS has been operated cryogenically with minimal thermal disturbance. A superconducting lead shield has been demonstrated to effectively shield external magnetic fields, and field gradients in the prototype are comparable or 


\begin{tabular}{cccc}
\hline$<d B_{x_{i}} / d x_{i}>/ B_{0}(\mathrm{ppm} / \mathrm{cm})$ & $\left\langle d B_{x} / d x>/ B_{0}\right.$ & $\left\langle d B_{y} / d y>/ B_{0}\right.$ & $<d B_{z} / d z>/ B_{0}$ \\
\hline Right Cell & 2.5 & -5.6 & 2.3 \\
Left Cell & -3.9 & 5.6 & -0.73 \\
\hline
\end{tabular}

Table 2: The best relative magnetic gradients measured in the prototype apparatus, with $B_{0}$ set to $30 \mu \mathrm{T}$. The gradient values have been adjusted to those appropriate for the full-scale experiment. The volume average gradient is taken for left and right fiducial volumes. Statistical uncertainties are negligible. Note that the superconducting endcap is not in the superconducting state for these measurements.

less than the $3 \mathrm{ppm} / \mathrm{cm}$ required by the full experiment. Additionally, a partially superconducting endcap has been investigated experimentally and found to be well modeled by simulation.

Based on these results, we are presently investigating an optimized magnetic design that incorporates superconducting endcaps in a shorter magnetic coil. In this optimized design, the long axis of the measurement cell is oriented perpendicular to the magnet coil axis. This is in contrast with the work presented in this paper, where the long axis is aligned parallel to the coil axis.

\section{Acknowledgments}

The authors thank R. Golub, M. Hayden, and S. Lamoreaux for their many useful comments and suggestions. This work was supported by U.S. National Science Foundation grants 1205977 and 1506459 . 


\section{References}

[1] A. Pérez Galván, et al. Nucl. Instr. Meth. Phys. Res. A, 660:147-153, 2011.

[2] N. Ramsey. Rep. Prog. Phys, 45(1):95-113, 1982.

[3] A. D. Sakharov. JETP Lett., 5:24, 1967.

[4] T. Chupp and M. Ramsey-Musolf. Phys. Rev. C, 91:035502, 2015.

[5] M. Jung and A. Pich. JHEP, 76, 2014.

[6] S. Inoue, O. Ovanesyan, and M. Ramsey-Musolf. Phys. Rev. D, 93:015013, 2016.

[7] J. M. Pendlebury, et al. Phys. Rev. D, 92:092003, 2015.

[8] S. Clayton. Search for an EDM of the neutron at the SNS. In 5th International Symposium on Lepton Moments, 2014.

[9] R. Golub and S. Lamoreaux. Physics Reports, 237(1):1-62, 1994.

[10] J. M. Pendlebury, et al. Phys. Rev. A, 70:032102, 2004.

[11] R. Golub and J. M. Pendlebury. Phys. Lett., 53A:133, 1975.

[12] R. Golub and J. M. Pendlebury. Phys. Lett., 62A:337, 1977.

[13] K. P. Coulter, et al. NIM A, (288):463-466, 1990.

[14] V. A. Dzuba, V. V. Flambaum, and J. S. M. Ginges. Phys. Rev. A, 76:034501, 2007.

[15] G. Pignol, M. Guigue, A. K. Petukhov, and R. Golub. Phys. Rev. A, 92:053407, 2015.

[16] C. M. Swank, A. K Petukhov, and R. Golub. Random walks with thermalizing collisions in bounded regions: Physical applications valid from the ballistic to diffusive regimes. Phys. Rev. A, 93:062703, 2016.

[17] J. D Jackson. Classical Electrodynamics. John Wiley and Sons, Inc., 3rd edition, 1999, p 232, Problem 5.30.

[18] National Instruments ${ }^{\mathrm{TM}}$. http://www.ni.com/labview/.

[19] Measurement Computing Corporation. http://www.mccdaq.com/.

[20] Metglas, Inc. http://www.metglas.com.

[21] Prime Conduit, Cleveland, OH, USA. http://www.primeconduit.com/vylon-pipe/.

[22] S. Malkowski, et al. Rev. Sci. Instrum., 82:075104, 2011.

[23] AMETEK Programmable Power, Inc. http://www.programmablepower.com/brands/california-instruments.htm.

[24] Bartington Instruments, Ltd. http://www.bartington.com.

[25] National Instruments ${ }^{\mathrm{TM}}$. http://www.ni.com/.

[26] Dip Braze, Inc. http://www.dipbraze.com.

[27] Interface Welding. Carson, CA, USA. http://www.interfacewelding.com.

[28] Swagelok Company. https://www.swagelok.com/downloads/webcatalogs/EN/MS-01-24.PDF.

[29] Alicat Scientific, Inc. http://www.alicat.com/.

[30] Lake Shore Cryotronics, Inc. www.lakeshore.com.

[31] S. Fahy, C. Kittel, and S. G. Louie. Am. J. Phys., 56(11):989-992, 1988.

[32] COMSOL Multiphysics, v. 5.2. www.comsol.com. COMSOL AB, Stockholm, Sweden.

[33] J. D. Jackson. Classical Electrodynamics. John Wiley and Sons, Inc., 3rd edition, 1999, p 205. 